\title{
Formation of Galactic Nuclei by Globular Cluster Merging
}

\author{
P. Miocchi and R. Capuzzo Dolcetta \\ Department of Physics, Universitá di Roma "La Sapienza", P.le A. Moro, 5, I-00185, Italy \\ email: miocchi,roberto.capuzzodolcetta@uniroma1.it
}

\begin{abstract}
Recent HST observations have revealed that compact sources exist at the centers of many galaxies across the Hubble sequence. These sources are called "nuclear star clusters" (NCs), because their structural properties and scaling relationships are similar to those of globular clusters (GCs). It has been also found that the relationship between the masses of NCs and that of the host galaxies is similar to that obeyed by supermassive black holes (SBHs). In this observational frame, the hypothesis that galactic nuclei may be the remains of GCs driven inward to the galactic center by dynamical friction and there merged, finds an exciting possible confirm. In this short paper we report of our recent results on GC mergers obtained by mean of detailed $N$-body simulations.
\end{abstract}

Keywords. stellar dynamics, methods: n-body simulations, globular clusters: general, galaxies: nuclei, galaxies: kinematics and dynamics, galaxies: star clusters

\section{Introduction}

In many early-type galaxies (Côté et al. 2006) and late-type spirals (Böker et al. 2002, Rossa et al. 2006) evident compact nuclei have been resolved, showing a luminosity distribution much better fitted by an extended (King's) profile rather than by a point source (Fig. 1). Such compact nuclei are likely the low-mass counterparts of nuclei hosting SBHs detected in bright galaxies (see also Wehner \& Harris 2006). It is clear that these characteristics of galaxies nuclei well fit into a "dissipationless" scenario of multiple GCs merging in the inner galactic regions. So far, not many $N$-body simulations have been presented in the literature that study the dissipationless formation of NCs. Among these, we remind those by Fellhauer \& Kroupa (2005) finding that super-massive star clusters, like W3 in NGC 7252, are very likely the merging remnants of smaller systems. Also Bekki et al. (2004) examined the merger formation scenario of NCs, though with simulations done in a simplified way, neglecting the role of the galactic external potential and with a relatively low resolution.

\section{The Simulations}

Here we report briefly of some of the results presented in Capuzzo-Dolcetta \& Miocchi (2007b, in preparation), where we studied whether and how the merging of various massive GCs decayed by dynamical friction in the inner galactic region may occur. These results give substance to the interpretation of the formation of galaxies nuclei via merging of decayed GCs (see Capuzzo Dolcetta \& Vicari (2005) and references therein). We consider GCs as $N$-body systems moving within a triaxial galaxy represented by an analytical potential, subjected also to dynamical friction. We studied the merging process occurring among four GCs already decayed within $100 \mathrm{pc}$ from the galactic center. The galaxy where the GCs move is represented by a self-consistent triaxial potential. Simulations are done with the parallel 'ATD' $N$-body code (Miocchi \& Capuzzo Dolcetta, 

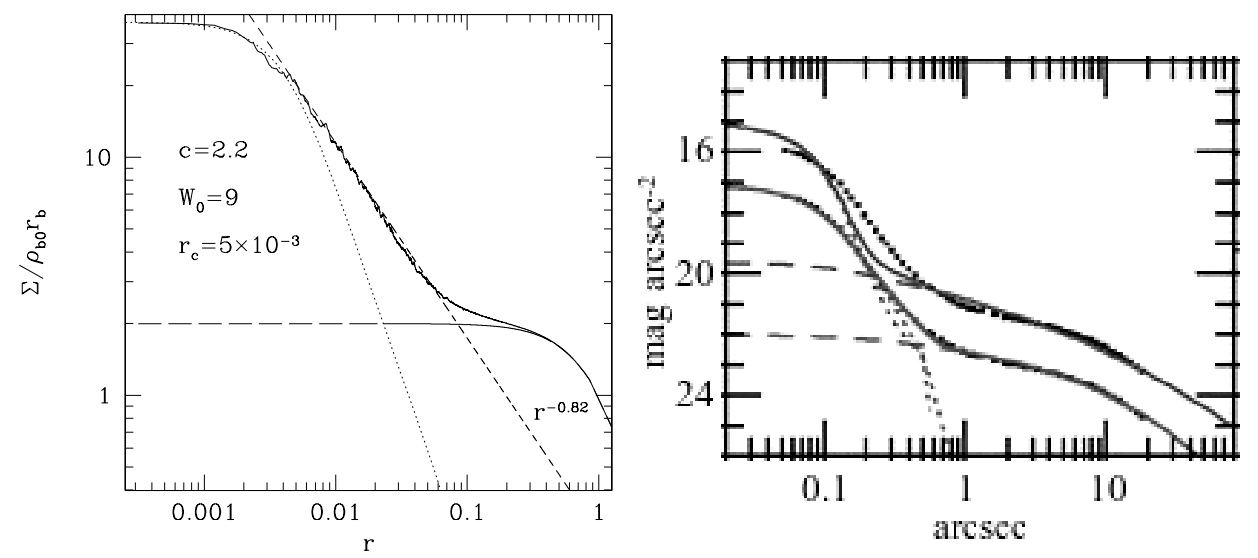

Figure 1. Left panel: Projected surface density profile for the last NC configuration (in proper units), overlapped to the galaxy background profile (long dashed line) so to give the total surface density (solid line). The short dashed line indicates the $r^{-0.82}$ behaviour, while the dotted one is the core best-fit King profile whose parameters are also reported. Right panel: surface brightness profile of VCC 1661 (black squares); the lower solid line is the superposition of a fitting Sérsic model for the galaxy (long dashed) and a King model for the core region (dotted); the upper solid line is the fitting with a point source instead of the King model. The two curves are translated for display convenience. From Côté et al. 2006.

2002), with a total of $10^{6}$ particles. See Capuzzo-Dolcetta \& Miocchi (2007a) for more details.

\section{Results}

After $\sim 15$ Myr, corresponding to $\sim 20$ galactic core crossing times, the merging is completed and the resulting system attains a quasi-equilibrium configuration. This gives a total projected density profile in the nuclear region that is remarkably similar to those recently observed in the central regions of nucleated early-type galaxies in the Virgo cluster (Fig. 1), as well as in nearby late-type spirals. The final NC morphology is that of an axisymmetric ellipsoid (axial ratios 1.4:1.4:1, ellipticity $\sim 0.3$ ) without figure rotation. In the velocity dispersion-mass plane, the NC is located closer to the scaling relation followed by GCs than to that of elliptical galaxies.

\section{References}

Bekki, K., Couch, W. J., Drinkwater, M. J. \& Shioya, Y. 2004, ApJL 610, L13

Böker, T., Laine, S., van der Marel, R. P., Sarzi, M., Rix, H. -W., Ho, L. C. \& Shields, J. C. 2002, AJ, 123, 1389

Capuzzo Dolcetta, R. \& Miocchi, P. 2007a, in: J. Knapen, T. Mahoney \& A. Vazdekis (eds.), Pathways Through an Eclectic Universe (San Francisco: ASP), in press (astro$\mathrm{ph} / 0709.0455)$

Capuzzo-Dolcetta, R. \& Vicari, A. 2005, MNRAS 356, 899

Côté, P. et al. 2006, ApJS 165, 57

Fellhauer, M. \& Kroupa, P. 2005, MNRAS 359, 223

Miocchi, P. \& Capuzzo Dolcetta, R. 2002, A\&A 382, 758

Rossa, J., van der Marel, R.P., Boeker, T., Gerssen, J., Ho, L.C., et al. 2006, AJ 132, 1074

Wehner, E. H. \& Harris, W. 2006, ApJL 644, L17 